\title{
A convenient synthesis of novel substituted imidazo[1,2-a][1,5]benzodiazepine derivatives
}

\author{
Lidija Kosychova,* Lina Pleckaitiene, Zita Staniulyte, Regina Janciene, Algirdas Palaima, \\ and Benedikta D. Puodziunaite
}

Institute of Biochemistry, Mokslininku 12, LT-08662 Vilnius, Lithuania

E-mail: kosychova@bchi.lt

\begin{abstract}
The synthesis of 2-substituted imidazo[1,2-a][1,5]benzodiazepines is accomplished from tetrahydro-1,5-benzodiazepin-2-ones and $\alpha$-haloketones. The structure and stereochemistry of the ring system obtained were investigated by ${ }^{1} \mathrm{H}$ and ${ }^{13} \mathrm{C}$ NMR spectroscopy.
\end{abstract}

Keywords: Imidazo[1,2-a][1,5]benzodiazepine, tetrahydro-1,5-benzodiazepin-2-ones, $\quad \alpha$ haloketones, alkylation, phase-transfer catalysis, cyclization

\section{Introduction}

Benzodiazepines and their polycyclic derivatives are known to exhibit a wide spectrum of biological activities and have found applications in the pharmaceutical chemistry. ${ }^{1,2}$ Moreover, a considerable amount of attention has been focused on the preparation of various tricyclic imidazo[1,4] and [1,5]benzodiazepines. ${ }^{2,3-10}$ Recently, we have reported that several members of peri-annelated imidazo[1,5]benzodiazepine derivatives showed in vitro anti-HIV activity. ${ }^{11}$ With the aim of expanding our research on this heterocyclic ring system we have been interested in the synthesis of compounds containing an imidazole nucleus fused at 1 and 2 positions of 1,5benzodiazepine skeleton. In the present work we describe a new synthesis of 2-alkyl(or aryl)substituted imidazo[1,2-a][1,5]benzodiazepines from tetrahydro-1,5-benzodiazepin-2-one derivatives, bearing N-(2-oxo-propyl) and N-(2-oxo-2-phenyl-ethyl) groups, which were used as intermediates. Mostly, for the formation of imidazo[1,2- $a$ ] fused ring the interaction of the aminobenzodiazepines with propargylamine or with $\alpha$-halogen ketones ${ }^{5-7}$ has been employed.

\section{Results and Discussion}

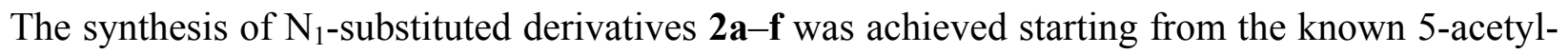
1,5-benzodiazepinones $\mathbf{1 a}-\mathbf{c}^{12}$ (Scheme 1). Compounds $\mathbf{2 a - f}$ could be synthesized by two 
methods. The direct reaction between lactams $1 \mathbf{a}-\mathbf{c}$ and twice excess of the chloroacetone at room temperature in the dry acetone / $\mathrm{KOH}$ system gave rise to $N$-(propyl-2'-oxo)-derivatives 2a-c (Method A). The reaction was exothermic and its outset was accompanied by an extensive darkening of the reaction mixture. Compounds $\mathbf{2 d - f}$ were obtained from $\mathbf{1 a}-\mathbf{c}$ and 2bromoacetophenone according to this procedure only at higher $35-40{ }^{\circ} \mathrm{C}$ temperature. Alternatively (Method B), lactams 1a-c were treated with chloroacetone or 2bromoacetophenone using the phase-transfer catalysis conditions to give compounds $\mathbf{2 a - \mathbf { f }}$ in good yields. The cyclodehydration of $\mathrm{N}_{1}$-substituted derivatives $\mathbf{2 a}-\mathbf{f}$ was performed by refluxing with ammonium acetate in glacial acetic acid and the desired tricyclic compounds $\mathbf{3 a}-\mathbf{f}$ were obtained.
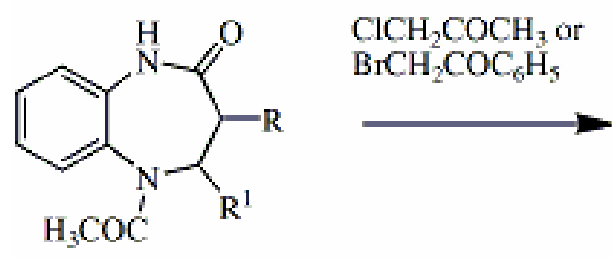

1a-c

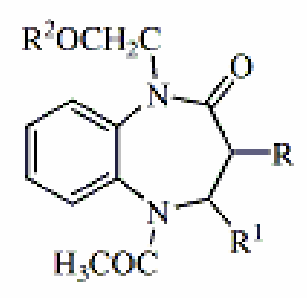

$2 a-f$

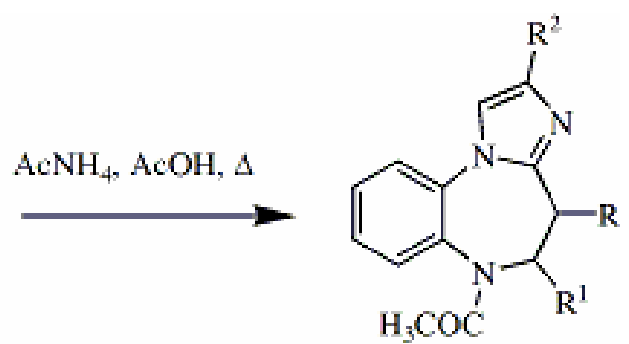

3a-f

$\begin{array}{llll} & \mathrm{R} & \mathrm{R}^{1} & \mathrm{R}^{2} \\ \text { a } & \mathrm{H} & \mathrm{H} & \mathrm{CH}_{3} \\ \text { b } & \mathrm{CH}_{3} & \mathrm{H} & \mathrm{CH}_{3} \\ \text { c } & \mathrm{H} & \mathrm{CH}_{3} & \mathrm{CH}_{3} \\ \text { d } & \mathrm{H} & \mathrm{H} & \mathrm{C}_{6} \mathrm{H}_{5} \\ \text { e } & \mathrm{CH}_{3} & \mathrm{H} & \mathrm{C}_{6} \mathrm{H}_{5} \\ \text { f } & \mathrm{H} & \mathrm{CH}_{3} & \mathrm{C}_{6} \mathrm{H}_{5}\end{array}$

\section{Scheme 1}

The purity and structures of the newly synthesized compounds were confirmed by elemental analysis and spectroscopic data, IR, ${ }^{1} \mathrm{H}$ and ${ }^{13} \mathrm{C}$ NMR. NMR signals assignment is supported by ${ }^{1} \mathrm{H}-{ }^{1} \mathrm{H}$ COSY and ${ }^{1} \mathrm{H},{ }^{13} \mathrm{C}$ shift correlation experiments. The ${ }^{1} \mathrm{H}$ NMR spectra of 3a-f showed characteristic signals for imidazo ring system - the quartet at 6.87-6.89 ppm $(J=$ $1.0 \mathrm{~Hz})(\mathbf{3 a}-\mathbf{c})$ and the singlet at 7.41-7.45 ppm (3d-f) for $\mathrm{H}-1$ and the doublet at 2.25-2.35 for 2- $\mathrm{CH}_{3}$ group (3a-c). The ABMX spin system of $\mathrm{CH}_{2} \mathrm{CH}_{2}$ moiety in ${ }^{1} \mathrm{H} \mathrm{NMR}$ spectra of $\mathbf{2 a , d}$ and 3a,d compounds clearly suggests only one conformer which does not interconvert at room temperature. The values $(12.5$ to $12.8 \mathrm{~Hz}$ ) of vicinal coupling constants of methine protons (5- or 4- $\mathrm{CH}$ ) and one proton of methylene groups (4- or 5- $\mathrm{CH}_{2}$ ) for compounds $\mathbf{3 b}$,c,e,f showed antiperiplanar arrangement of these protons, i.e., the conformational equilibrium at the seven membered ring in tricyclic benzodiazepines was shifted towards the direction where the methyl group was quasi equatorial, analogously as in the precursors $\mathbf{2 b , c , e , f . ~ I n ~ t h e ~}{ }^{13} \mathrm{C} N M R$ spectra of 3a-f the C-4, C-3a and C-10a resonances were mainly influenced by replacement of the lactam 
functionality with imidazole nucleus and were shifted upfield (about 6-8, 25 and 5 ppm, respectively) with respect to the precursors $\mathbf{2 a}-\mathbf{f}$. In addition, the $\mathrm{C}-1$ resonances of the imidazole ring were observed at $113.9-114.2$ ppm with ${ }^{1} J_{\mathrm{CH}} 188.8$ to $189.3 \mathrm{~Hz}$.

\section{Experimental Section}

General Procedures. Melting points were determined in open capillaries and are uncorrected. IR spectra were taken on a Perkin Elmer Spectrum GX FT-IR spectrometer in KBr tablets. ${ }^{1} \mathrm{H}$ (300 MHz) and ${ }^{13} \mathrm{C}(75 \mathrm{MHz})$ NMR spectra were recorded on a Varian Unity Inova 300 spectrometer at $302 \mathrm{~K}$ in deuteriochloroform with TMS as an internal standard. The values of chemical shifts are expressed in ppm and coupling constants $(J)$ in $\mathrm{Hz}$. The $\mathrm{CH}_{3}, \mathrm{CH}_{2}, \mathrm{CH}$ and $\mathrm{C}_{\text {quaternary }}$ groups in ${ }^{13} \mathrm{C}$ NMR were differentiated by means of the APT method. The reactions were controlled by TLC performed on Silufol $\mathrm{UV}_{254}$ silica gel plates in diethyl ether-benzenemethanol ( $v / v, 6: 1: 1)$ (compounds 2a-f) and butanol-acetic acid-water( $v / v, 4: 2: 1$ ) (compounds 3a-f) used as eluents. Elemental analysis for $\mathrm{C}, \mathrm{H}, \mathrm{N}$ was performed on the Microelemental analyzer (Labopribor). Elemental analysis data of all new compounds agreed with the theoretical values to within $\pm 0,4 \%$.

General procedure for the synthesis of 5-acetyl-4-R ${ }^{1}-3-R-1-(2-o x o-p r o p y l)-1,3,4,5-$ tetrahydro-2H-1,5-benzodiazepin-2-one $(2 \mathrm{a}-\mathrm{c})$ and 5 -acetyl-4-R ${ }^{\mathbf{1}}-\mathbf{3}-\mathrm{R}-\mathbf{1}-(\mathbf{2}-\mathrm{oxo}-\mathbf{2}-\mathrm{phenyl-}$ ethyl)-1,3,4,5-tetrahydro-2H-1,5-benzodiazepin-2-one (2d-f).

Method A. To a stirred suspension of a suitable acetylbenzodiazepinone derivative 1a-c (4.5 $\mathrm{mmol})$, finely powdered potassium hydroxide $(1.26 \mathrm{~g}, 22.5 \mathrm{mmol})$ in dry acetone $(40 \mathrm{ml})$, a precooled solution of chloroacetone $(0.73 \mathrm{ml}, 9.0 \mathrm{mmol})$ in dry acetone $(10 \mathrm{ml})$ or solution of 2bromoacetophenone $(1.79 \mathrm{~g}, 9.0 \mathrm{mmol})$ in dry acetone $(60 \mathrm{ml})$ was added dropwise $(15 \mathrm{~min}$.) at room temperature. After the initial exothermic reaction the mixture with the chloroacetone was stirred at room temperature and then filtered. In each case the optimum reaction time (ca 1,5-3 h) was determined by TLC monitoring. The reaction mixture with the 2-bromoacetophenone was heated to $35-40{ }^{\circ} \mathrm{C}$ and was kept at this temperature under stirring for 40-50 min., then cooled and filtered. The filtrate was evaporated in vacuum to a dark oily residue. The product was dissolved in chloroform $(70 \mathrm{ml})$, washed with brine $(2 \times 40 \mathrm{ml})$, organic layer was dried $\left(\mathrm{MgSO}_{4}\right)$ and concentrated to dryness in vacuum. Compounds $\mathbf{2 a - f}$ were obtained as white crystals by recrystallization from a proper solvent.

Method B. To a stirred solution of the benzodiazepinone derivative 1a-c (10 mmol), benzyltriethylamonium chloride $(1.13 \mathrm{~g}, 5 \mathrm{mmol})$, chloroacetone $(1.2 \mathrm{ml}, 15 \mathrm{mmol})$ in acetonitrile $(60 \mathrm{ml})$, finely powdered potassium carbonate $(3.5 \mathrm{~g}, 25 \mathrm{mmol})$ was added. The reaction mixture was refluxed under stirring for $7 \mathrm{~h}$, allowed to cool and filtered. The filtrate was evaporated in vacuum and the residue taken up with chloroform $(100 \mathrm{ml})$. The organic solution was washed with brine $(3 \times 25 \mathrm{ml})$, organic layer was dried over $\mathrm{MgSO}_{4}$ and the solvent was 
removed in vacuum. The crude product $\mathbf{2 a - c}$ was crystallized by adding small amounts of diethyl ether or ethyl acetate.

Compounds $\mathbf{2 d - f}$ were prepared according to the same procedure using 2-bromoacetophenone (2.98 g, $15 \mathrm{mmol})$.

5-Acetyl-1-(2-oxo-propyl)-1,3,4,5-tetrahydro-2H-1,5-benzodiazepin-2-one (2a). White crystals, yield: $30 \%$ (method A), $60 \%$ (method B), m.p.: $149-152{ }^{\circ} \mathrm{C}$ (diethyl ether). IR $\left(\mathrm{cm}^{-1}\right)$ : 1731, 1658. ${ }^{1} \mathrm{H}$ NMR $\left(\mathrm{CDCl}_{3}\right) \delta: 1.90\left(3 \mathrm{H}, \mathrm{s}, 5-\mathrm{CH}_{3}\right), 2.21\left(3 \mathrm{H}, \mathrm{s}, \mathrm{CH}_{3}\right), 2.49\left(1 \mathrm{H}, \mathrm{m}, \mathrm{CH}_{2}\right), 2.66$ $\left(1 \mathrm{H}, \mathrm{m}, \mathrm{CH}_{2}\right), 3.51\left(1 \mathrm{H}, \mathrm{m}, 4-\mathrm{CH}_{2}\right), 4.50$ and $4.64\left(2 \mathrm{H}, \mathrm{AB}-\mathrm{q}, J=17.8 \mathrm{~Hz}, 1-\mathrm{CH}_{2}\right), 4.94(1 \mathrm{H}, \mathrm{m}$, 4- $\left.\mathrm{CH}_{2}\right), 7.17(1 \mathrm{H}, \mathrm{dd}, J=1.4,7.9 \mathrm{~Hz}, \mathrm{H}-6$ or H-9), $7.21(1 \mathrm{H}, \mathrm{dd}, J=1.6,7.8 \mathrm{~Hz}, \mathrm{H}-9$ or H-6), $7.30(1 \mathrm{H}, \mathrm{dt}, J=1.5,7.8 \mathrm{~Hz}, \mathrm{H}-7$ or $\mathrm{H}-8), 7.40\left(1 \mathrm{H}, \mathrm{dt}, J=1.6,7.8 \mathrm{~Hz}, \mathrm{H}-8\right.$ or H-7). ${ }^{13} \mathrm{C}$ NMR $\left(\mathrm{CDCl}_{3}\right)$ \&: $22.76\left(5-\mathrm{CH}_{3}\right), 27.15\left(1-\mathrm{CH}_{3}\right), 32.98(\mathrm{C}-3), 47.87(\mathrm{C}-4), 57.18\left(1-\mathrm{CH}_{2}\right), 123.06$, 127.14, 129.35, 129.82, 134.79 (C-5a), 140.42 (C-9a), 170.48 (5-CO), 170.89 (C-2), 201.66 (1$\mathrm{CO}$ ). Anal. Calcd. for $\mathrm{C}_{14} \mathrm{H}_{16} \mathrm{~N}_{2} \mathrm{O}_{3}$ (260.30): C, 64.60; H, 6.20; N, 10.76. Found: C, 64.78; H, $6.22 ; \mathrm{N}, 10.79 \%$.

5-Acetyl-3-methyl-1-(2-oxo-propyl)-1,3,4,5-tetrahydro-2H-1,5-benzodiazepin-2-one (2b). White crystals, yield: $42 \%(\operatorname{method} A), 59 \%(\operatorname{method} \mathrm{B}), \mathrm{m} . \mathrm{p} .: 121-123{ }^{\circ} \mathrm{C}$ (diethyl ether). IR $\left(\mathrm{cm}^{-1}\right): 1733,1662 .{ }^{1} \mathrm{H} \mathrm{NMR}\left(\mathrm{CDCl}_{3}\right) \delta: 1.11\left(3 \mathrm{H}, \mathrm{d}, J=6.5 \mathrm{~Hz}, \mathrm{CH}_{3}\right), 1.89\left(3 \mathrm{H}, \mathrm{s}, 5-\mathrm{CH}_{3}\right), 2.21$ $\left(3 \mathrm{H}, \mathrm{s}, \mathrm{CH}_{3}\right), 2.76(1 \mathrm{H}, \mathrm{m}, \mathrm{CH}), 3.45\left(1 \mathrm{H}, \mathrm{dd}, J=6.4,12.7 \mathrm{~Hz}, \mathrm{CH}_{2}\right), 4.49$ and $4.63(2 \mathrm{H}, \mathrm{AB}-\mathrm{q}$, $\left.J=17.7 \mathrm{~Hz}, 1-\mathrm{CH}_{2}\right), 4.56\left(1 \mathrm{H}, \mathrm{dd}, J=13.0,13.0 \mathrm{~Hz}, \mathrm{CH}_{2}\right), 7.18(1 \mathrm{H}, \mathrm{dd}, J=1.5,7.9 \mathrm{~Hz}, \mathrm{H}-6$ or H-9), 7.21 (1H, dd, $J=1.6,7.8 \mathrm{~Hz}, \mathrm{H}-9$ or H-6) $7.29(1 \mathrm{H}, \mathrm{dt}, J=1.5,7.8 \mathrm{~Hz}, \mathrm{H}-7$ or H-8), 7.40 $(1 \mathrm{H}, \mathrm{dt}, J=1.6,7.8 \mathrm{~Hz}, \mathrm{H}-8$ or $\mathrm{H}-7) .{ }^{13} \mathrm{C} \mathrm{NMR}\left(\mathrm{CDCl}_{3}\right) \delta: 12.74\left(3-\mathrm{CH}_{3}\right), 22.66\left(5-\mathrm{CH}_{3}\right), 27.18$ $\left(1-\mathrm{CH}_{3}\right), 35.33(\mathrm{C}-3), 54.91(\mathrm{C}-4), 57.55\left(1-\mathrm{CH}_{2}\right), 123.25,127.04,129.32,129.42,135.43(\mathrm{C}-$ 5a), 140.02 (C-9a), 170.28 (5-CO), 173.15(C-2), 201.90 (1-CO). Anal. Calcd. for $\mathrm{C}_{15} \mathrm{H}_{18} \mathrm{~N}_{2} \mathrm{O}_{3}$ (274.32): C, 65.68; H, 6.61; N, 10.21. Found: C, 65.84; H, 6.62; N, 10.19\%.

5-Acetyl-4-methyl-1-(2-oxo-propyl)-1,3,4,5-tetrahydro-2H-1,5-benzodiazepin-2-one (2c). White crystals, yield: $50 \%(\operatorname{method} A), 44 \%(\operatorname{method} \mathrm{B}), \mathrm{m} . \mathrm{p} .: 154-156{ }^{\circ} \mathrm{C}$ (diethyl ether). IR $\left(\mathrm{cm}^{-1}\right): 1732,1658 .{ }^{1} \mathrm{H} \mathrm{NMR}\left(\mathrm{CDCl}_{3}\right) \delta: 1.16\left(3 \mathrm{H}, \mathrm{d}, J=6.3 \mathrm{~Hz}, \mathrm{CH}_{3}\right), 1.84\left(3 \mathrm{H}, \mathrm{s}, 5-\mathrm{CH}_{3}\right), 2.21$ $\left(3 \mathrm{H}, \mathrm{s}, \mathrm{CH}_{3}\right), 2.31\left(1 \mathrm{H}, \mathrm{dd}, J=13.0,13.0 \mathrm{~Hz}, \mathrm{CH}_{2}\right), 2.46\left(1 \mathrm{H}, \mathrm{dd}, J=5.2,13.0 \mathrm{~Hz}, \mathrm{CH}_{2}\right), 4.47$ and $4.63\left(2 \mathrm{H}, \mathrm{AB}-\mathrm{q}, J=17.7 \mathrm{~Hz}, 1-\mathrm{CH}_{2}\right), 5.31(1 \mathrm{H}, \mathrm{m}, \mathrm{CH}), 7.16(1 \mathrm{H}, \mathrm{dd}, J=1.5,7.6 \mathrm{~Hz}, \mathrm{H}-6$ or H-9), $7.17(1 \mathrm{H}, \mathrm{dd}, J=1.6,7.7 \mathrm{~Hz}, \mathrm{H}-9$ or H-6), $7.30(1 \mathrm{H}, \mathrm{dt}, J=1.5,7.8 \mathrm{~Hz}, \mathrm{H}-7$ or H-8), $7.42(1 \mathrm{H}, \mathrm{dt}, J=1.6,7.8 \mathrm{~Hz}, \mathrm{H}-8$ or $\mathrm{H}-7) ;{ }^{13} \mathrm{C} \mathrm{NMR}\left(\mathrm{CDCl}_{3}\right) \delta: 18.85\left(4-\mathrm{CH}_{3}\right), 22.96\left(5-\mathrm{CH}_{3}\right)$, $27.16\left(1-\mathrm{CH}_{3}\right), 40.48(\mathrm{C}-3), 54.23(\mathrm{C}-4), 57.04\left(1-\mathrm{CH}_{2}\right), 122.93,126.85,129.45,131.04,133.00$ (C-5a), 140.79 (C-9a), 169.60 (5-CO), 170.54 (C-2), 201.69 (1-CO). Anal. Calcd. for $\mathrm{C}_{15} \mathrm{H}_{18} \mathrm{~N}_{2} \mathrm{O}_{3}$ (274.32): C, 65.68; H, 6.61; N, 10.21. Found: C, 65.62; H, 6.59; N, $10.23 \%$.

\section{5-Acetyl-1-(2-oxo-2-phenyl-ethyl)-1,3,4,5-tetrahydro-2H-1,5-benzodiazepin-2-one}

(2d). White crystals, yield: $52 \%(\operatorname{method} A), 62 \%(\operatorname{method} B)$, m.p.: $200-201{ }^{\circ} \mathrm{C}$ (ethyl acetate). IR $\left(\mathrm{cm}^{-1}\right): 1703,1658 .{ }^{1} \mathrm{H} \mathrm{NMR}\left(\mathrm{CDCl}_{3}\right) \delta: 1.86\left(3 \mathrm{H}, \mathrm{s}, \mathrm{CH}_{3}\right), 2.55\left(1 \mathrm{H}, \mathrm{m}, \mathrm{CH}_{2}\right), 2.74(1 \mathrm{H}, \mathrm{m}$, $\left.\mathrm{CH}_{2}\right), 3.54\left(1 \mathrm{H}, \mathrm{m}, 4-\mathrm{CH}_{2}\right), 4.98\left(1 \mathrm{H}, \mathrm{m}, 4-\mathrm{CH}_{2}\right), 5.22$ and $5.27\left(2 \mathrm{H}, \mathrm{AB}-\mathrm{q}, J=17.5 \mathrm{~Hz}, 1-\mathrm{CH}_{2}\right)$, $7.22(1 \mathrm{H}, \mathrm{dd}, J=1.4,7.9 \mathrm{~Hz}, \mathrm{H}-6$ or H-9), $7.25(1 \mathrm{H}, \mathrm{dd}, J=1.6,7.8 \mathrm{~Hz}, \mathrm{H}-9$ or H-6), 7.29 (1H, $\mathrm{dt}, J=1.5,7.8 \mathrm{~Hz}, \mathrm{H}-7$ or H-8), 7.39 (1H, dt, $J=1.7,7.8 \mathrm{~Hz}, \mathrm{H}-8$ or H-7), 7.48 (2H, m, H-3', H- 
$\left.5^{\prime}\right), 7.61(1 \mathrm{H}, \mathrm{m}, \mathrm{H}-4), 7.97\left(2 \mathrm{H}, \mathrm{m}, \mathrm{H}-2^{\prime}, 6^{\prime}\right) .{ }^{13} \mathrm{C} \mathrm{NMR}\left(\mathrm{CDCl}_{3}\right)$ 8: $22.72\left(5-\mathrm{CH}_{3}\right), 33.11(\mathrm{C}-3)$, $47.94(\mathrm{C}-4), 53.77\left(1-\mathrm{CH}_{2}\right), 123.06,127.03,128.01,128.00$ (C - 3', 5'), $128.78\left(\mathrm{C}-2^{\prime}, 6^{\prime}\right)$, 129.27, 129.82, 133.82 (C-4'), 134.61 (C-1'), 134.86 (C-5a), 140.35 (C-9a), 170.59 (5-CO), 170.99 (C-2), 192.90 (1- CO). Anal. Calcd. for $\mathrm{C}_{19} \mathrm{H}_{18} \mathrm{~N}_{2} \mathrm{O}_{3}$ (322.37): C, 70.79; H, 5.63; N, 8.69. Found: C, 70.96; H, 5.64; N, 8.71\%.

5-Acetyl-3-methyl-1-(2-oxo-2-phenyl-ethyl)-1,3,4,5-tetrahydro-2H-1,5-benzodiazepin-2-one (2e). White crystals, yield: $61 \%(\operatorname{method} A), 60 \%$ (method B), m.p.: 195-196 ${ }^{\circ} \mathrm{C}$ (ethyl acetate). IR $\left(\mathrm{cm}^{-1}\right)$ 1702, 1658. ${ }^{1} \mathrm{H} \mathrm{NMR}\left(\mathrm{CDCl}_{3}\right) \delta: 1.16\left(3 \mathrm{H}, \mathrm{d}, J=6.5 \mathrm{~Hz}, \mathrm{CH}_{3}\right), 1.85\left(3 \mathrm{H}, \mathrm{s}, 5-\mathrm{CH}_{3}\right)$, $2.85(1 \mathrm{H}, \mathrm{m}, \mathrm{CH}), 3.49\left(1 \mathrm{H}, \mathrm{dd}, J=6.3,12.7 \mathrm{~Hz}, \mathrm{CH}_{2}\right), 4.60\left(1 \mathrm{H}, \mathrm{dd}, J=12.7,13.0 \mathrm{~Hz}, \mathrm{CH}_{2}\right)$, 5.22 and $5.27\left(2 \mathrm{H}, \mathrm{AB}-\mathrm{q}, J=17.4 \mathrm{~Hz}, 1-\mathrm{CH}_{2}\right), 7.21-7.32(3 \mathrm{H}, \mathrm{m}, \mathrm{Ar}), 7.39(1 \mathrm{H}, \mathrm{dt}, J=1.7,7.8$ $\mathrm{Hz}, \mathrm{H}-8$ or H-7), 7.48 (2H, m, H-3', H-5'), 7.61 (1H, m, H-4'), 7.97 (2H, m, H-2', 6'). ${ }^{13} \mathrm{C}$ NMR

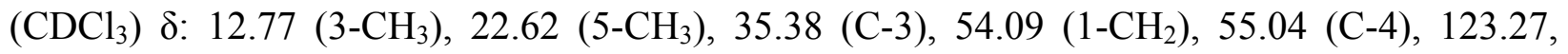
126.94, 128.04 (C-3',5'), 128.78 (C-2',6'), 129.26, $129.44,133.78$ (C-4'), 134.74 (C-1'), 135.54, (C-5a ), 139.98 (C-9a), 170.40 (5-CO), 173.24 (C-2), 193.24 (1-CO). Anal. Calcd. for $\mathrm{C}_{20} \mathrm{H}_{20} \mathrm{~N}_{2} \mathrm{O}_{3}$ (336.39): C, 71.41; H, 5.99; N, 8.33. Found: C, 71.38; H, 6.00; N, 8.31\%.

5-Acetyl-4-methyl-1-(2-oxo-2-phenyl-ethyl)-1,3,4,5-tetrahydro-2H-1,5-benzodiazepin-2-one (2f). White crystals, yield: $65 \%$ (method A), $75 \%$ (method B), m.p.: 191-193 ${ }^{\circ} \mathrm{C}$ (ethyl acetate). IR $\left(\mathrm{cm}^{-1}\right): 1708,1673,1642 .{ }^{1} \mathrm{H}$ NMR $\left(\mathrm{CDCl}_{3}\right) \delta: 1.20\left(3 \mathrm{H}, \mathrm{d}, J=6.3 \mathrm{~Hz}, \mathrm{CH}_{3}\right), 1.81(3 \mathrm{H}, \mathrm{s}, 5-$ $\left.\mathrm{CH}_{3}\right), 2.35-2.57\left(2 \mathrm{H}, \mathrm{m}, 3-\mathrm{CH}_{2}\right), 5.19$ and $5.28\left(2 \mathrm{H}, \mathrm{AB}-\mathrm{q}, J=17.5 \mathrm{~Hz}, 1-\mathrm{CH}_{2}\right), 5.35(1 \mathrm{H}, \mathrm{m}$, $\mathrm{CH}), 7.18(1 \mathrm{H}, \mathrm{dd}, J=1.5,7.8 \mathrm{~Hz}, \mathrm{H}-6$ or H-9), 7.25 (1H, dd, $J=1.5,7.8 \mathrm{~Hz}, \mathrm{H}-9$ or H-6), 7.30 $(1 \mathrm{H}, \mathrm{dt}, J=1.6,7.6 \mathrm{~Hz}, \mathrm{H}-7$ or H-8), $7.41(1 \mathrm{H}, \mathrm{dt}, J=1.5,7.6 \mathrm{~Hz}, \mathrm{H}-8$ or H-7), 7.49 (2H, m, H-

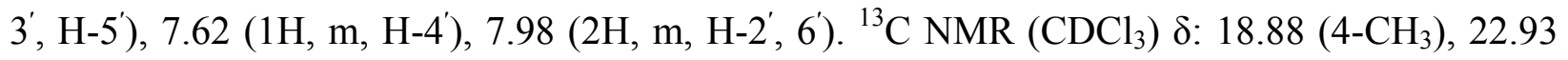
$\left(5-\mathrm{CH}_{3}\right), 40.64(\mathrm{C}-3), 53.64\left(1-\mathrm{CH}_{2}\right), 54.33(\mathrm{C}-4), 122.93,126.75,128.04\left(\mathrm{C}-3{ }^{\prime}, 5^{\prime}\right), 128.80(\mathrm{C}-$ 2',6'), 129.39, 131.05, 133.10 (C-5a), 133.82 (C-4'), 134.67 (C-1'), 140.76 (C-9a), 169.72 (5-CO), 170.66 (C-2), 192.92 (1-CO). Anal. Calcd. for $\mathrm{C}_{20} \mathrm{H}_{20} \mathrm{~N}_{2} \mathrm{O}_{3}$ (336.39): C, 71.41; H, 5.99; N, 8.33. Found: C, 71.62; H, 5.98; N, 8.35\%.

General procedure for the synthesis of 6-acetyl-2-methyl- or 2-phenyl-5,6-dihydro-4Himidazo[1,2-a][1,5]benzodiazepine (3a-f).

A mixture of $\mathrm{N}$-substituted derivative $\mathbf{2 a}-\mathbf{f}(5 \mathrm{mmol})$ and ammonium acetate $(3.85 \mathrm{~g}, 50 \mathrm{mmol})$ in glacial acetic acid $(30 \mathrm{ml})$ was refluxed for $6 \mathrm{~h}$ for $\mathbf{3 a}-\mathbf{c}$ and $10 \mathrm{~h}$ for $\mathbf{3 d}-\mathbf{f}$. After cooling the reaction mixture was poured into ice-water (ca $300 \mathrm{~g}$ ) and neutralized with $30 \%$ ammonium hydroxide solution. In the case of $\mathbf{3 d}-\mathbf{f}$, the formed precipitate was filtered, washed with water, dried and recrystallized from a suitable solvent. In other cases, the mixture was extracted with ethyl acetate $(3 \times 40 \mathrm{ml})$. The organic solution was washed with water $(2 \times 50 \mathrm{ml})$, dried over $\mathrm{MgSO}_{4}$, filtered and brought to dryness in vacuum. The residue was induced to crystallize by adding small amounts of diethyl ether.

6-Acetyl-2-methyl-5,6-dihydro-4H-imidazo[1,2-a][1,5]benzodiazepine (3a). White crystals, yield: $46 \%$, m.p.: $143-144{ }^{\circ} \mathrm{C}$ (diethyl ether). IR $\left(\mathrm{cm}^{-1}\right): 1659 .{ }^{1} \mathrm{H} \mathrm{NMR}\left(\mathrm{CDCl}_{3}\right) \delta: 1.71(3 \mathrm{H}, \mathrm{s}$, 6- $\left.\mathrm{CH}_{3}\right), 2.25\left(3 \mathrm{H}, \mathrm{d}, J=1.0 \mathrm{~Hz}, \mathrm{CH}_{3}\right), 2.73\left(1 \mathrm{H}, \mathrm{m}, \mathrm{CH}_{2}\right), 3.12\left(1 \mathrm{H}, \mathrm{m}, \mathrm{CH}_{2}\right), 3.54(1 \mathrm{H}, \mathrm{m}, 5-$ $\left.\mathrm{CH}_{2}\right), 4.96\left(1 \mathrm{H}, \mathrm{m}, 5-\mathrm{CH}_{2}\right), 6.88(1 \mathrm{H}, \mathrm{q}, J=1.0 \mathrm{~Hz}, 1-\mathrm{CH}), 7.31-7.42(3 \mathrm{H}, \mathrm{m}, \mathrm{Ar}), 7.47-7.53$ 
$\left(1 \mathrm{H}, \mathrm{m}, \mathrm{H}-8\right.$ or H-9). ${ }^{13} \mathrm{C} \mathrm{NMR}\left(\mathrm{CDCl}_{3}\right) \delta: 13.49\left(2-\mathrm{CH}_{3}\right), 22.82\left(6-\mathrm{CH}_{3}\right), 25.05(\mathrm{C}-4), 49.49(\mathrm{C}-$ 5), 114.17 (C-1), 123.23, 127.68, 129.63, 130.47, 134.19 (C-6a), 135.63 (C-10a ), 138.08 (C-2), 145.36 (C-3a), 170.48 (6-CO). Anal. Calcd. for $\mathrm{C}_{14} \mathrm{H}_{15} \mathrm{~N}_{3} \mathrm{O}$ (241.30): C, 69.69; H, 6.27; N, 17.41. Found: C, 69.56; H, 6.28; N, $17.44 \%$.

6-Acetyl-2,4-dimethyl-5,6-dihydro-4H-imidazo[1,2-a][1,5]benzodiazepine (3b). White crystals, yield: $54 \%$, m.p.: $160-162{ }^{\circ} \mathrm{C}$ (diethyl ether). IR $\left(\mathrm{cm}^{-1}\right): 1654 .{ }^{1} \mathrm{H} \mathrm{NMR}\left(\mathrm{CDCl}_{3}\right) \delta: 1.46$ $\left(3 \mathrm{H}, \mathrm{d}, J=6.8 \mathrm{~Hz}, \mathrm{CH}_{3}\right), 1.70\left(3 \mathrm{H}, \mathrm{s}, \mathrm{CH}_{3}\right), 2.29\left(3 \mathrm{H}, \mathrm{d}, J=1.0 \mathrm{~Hz}, \mathrm{CH}_{3}\right), 2.92(1 \mathrm{H}, \mathrm{m}, \mathrm{CH})$, $3.60\left(1 \mathrm{H}, \mathrm{dd}, J=6.1,12.5 \mathrm{~Hz}, \mathrm{CH}_{2}\right), 4.60\left(1 \mathrm{H}, \mathrm{dd}, J=12.7,12.7 \mathrm{~Hz}, \mathrm{CH}_{2}\right), 6.89(1 \mathrm{H}, \mathrm{q}, J=1.0$ $\mathrm{Hz}, 1-\mathrm{CH}), 7.31-7.43$ (3H, m Ar), $7.50(1 \mathrm{H}, \mathrm{m}, \mathrm{H}-8$ or $\mathrm{H}-9) .{ }^{13} \mathrm{C} \mathrm{NMR}\left(\mathrm{CDCl}_{3}\right) \delta$ : 13.60 (2$\left.\mathrm{CH}_{3}\right), 13.86\left(4-\mathrm{CH}_{3}\right), 22.75\left(6-\mathrm{CH}_{3}\right), 29.74(\mathrm{C}-4), 57.14(\mathrm{C}-5), 114.22(\mathrm{C}-1), 123.38,127.73$, 129.59, 130.17, 134.58 (C-6a), 135.49 (C-10a), 137.84 (C-2), 148.61 (C-3a), 170.44 (6-CO). Anal. Calcd. for $\mathrm{C}_{15} \mathrm{H}_{17} \mathrm{~N}_{3} \mathrm{O}$ (255.32): C, 70.56; H, 6.71; N, 16.46. Found: C, 70.44; H, 6.72; N, $16.51 \%$.

6-Acetyl-2,5-dimethyl-5,6-dihydro-4H-imidazo[1,2-a][1,5]benzodiazepine (3c). White crystals, yield: $61 \%$, m.p.: $142-144{ }^{\circ} \mathrm{C}$ (diethyl ether). IR $\left(\mathrm{cm}^{-1}\right): 1655 .{ }^{1} \mathrm{H}$ NMR $\left(\mathrm{CDCl}_{3}\right) \delta: 1.29$ $\left(3 \mathrm{H}, \mathrm{d}, J=6.3 \mathrm{~Hz}, \mathrm{CH}_{3}\right), 1.66\left(3 \mathrm{H}, \mathrm{s}, 6-\mathrm{CH}_{3}\right), 2.35\left(3 \mathrm{H}, \mathrm{d}, J=1.0 \mathrm{~Hz}, \mathrm{CH}_{3}\right), 2.37(1 \mathrm{H}, \mathrm{dd}, J=$ $\left.12.5,14.8 \mathrm{~Hz}, \mathrm{CH}_{2}\right), 3.09\left(1 \mathrm{H}, \mathrm{dd}, J=5.8,14.9 \mathrm{~Hz}, \mathrm{CH}_{2}\right), 5.33(1 \mathrm{H}, \mathrm{m}, \mathrm{CH}), 6.87(1 \mathrm{H}, \mathrm{q}, J=1.0$ $\mathrm{Hz}, 1-\mathrm{CH}), 7.26-7.30$ (1H, m, Ar), 7.38-7.44 (2H, m, Ar), 7.52 (1H, m, H-8 or H-9). ${ }^{13} \mathrm{C}$ NMR $\left(\mathrm{CDCl}_{3}\right) \delta: 13.49\left(2-\mathrm{CH}_{3}\right), 19.06\left(5-\mathrm{CH}_{3}\right), 23.06\left(6-\mathrm{CH}_{3}\right), 32.57(\mathrm{C}-4), 56.38(\mathrm{C}-5), 113.92(\mathrm{C}-1)$, 123.21, 127.60, 129.77, 131.79, 132.35 (C-6a), 136.01 (C-10a), 137.95 (C-2), 145.48 (C-3a), 169.78 (6-CO). Anal. Calcd. for $\mathrm{C}_{15} \mathrm{H}_{17} \mathrm{~N}_{3} \mathrm{O}$ (255.32): C, 70.56; H, 6.71; N, 16.46. Found: C, $70.62 ; \mathrm{H}, 6.69 ; \mathrm{N}, 16.50 \%$.

6-Acetyl-2-phenyl-5,6-dihydro-4H-imidazo[1,2-a][1,5]benzodiazepine (3d). White crystals, yield: $66 \%$, m.p.: $123-124{ }^{\circ} \mathrm{C}$ (cyclohexane). IR $\left(\mathrm{cm}^{-1}\right): 1659 .{ }^{1} \mathrm{H} \mathrm{NMR}\left(\mathrm{CDCl}_{3}\right) \delta: 1.86(3 \mathrm{H}, \mathrm{s}$, $\left.\mathrm{CH}_{3}\right), 2.80\left(1 \mathrm{H}, \mathrm{m}, \mathrm{CH}_{2}\right), 3.28\left(1 \mathrm{H}, \mathrm{m}, \mathrm{CH}_{2}\right), 3.59\left(1 \mathrm{H}, \mathrm{m}, 5-\mathrm{CH}_{2}\right), 5.02\left(1 \mathrm{H}, \mathrm{m}, 5-\mathrm{CH}_{2}\right), 7.24-$ $7.56(7 \mathrm{H}, \mathrm{m}, \mathrm{Ar}), 7.45(1 \mathrm{H}, \mathrm{s}, 1-\mathrm{CH}), 7.82(2 \mathrm{H}, \mathrm{m}, \mathrm{Ar}) .{ }^{13} \mathrm{C} \mathrm{NMR}\left(\mathrm{CDCl}_{3}\right)$ 8: $22.81\left(6-\mathrm{CH}_{3}\right)$, 24.98 (C-4), 49.37 (C-5), 113.37 (C-1), 123.37, 124.91 (C-2', 6'), 127.14 (C-4'), 128.24, 128.60 (C-3', 5'), 129.80, 130.57, 133.27 (C-1'), 134.28(C-6a ), 135.28 (C-10a ), 141.79 (C-2), 146.50 (C-3a), 170.55 (6-CO). Anal. Calcd. for $\mathrm{C}_{19} \mathrm{H}_{17} \mathrm{~N}_{3} \mathrm{O}$ (303.37): C, 75.23; H, 5.65; N, 13.85. Found: C, 75.25; H, 5.64; N, $13.87 \%$.

6-Acetyl-4-methyl-2-phenyl-5,6-dihydro-4H-imidazo[1,2-a][1,5]benzodiazepine (3e). White crystals, yield: $65 \%$, m.p.: 82-84 ${ }^{\circ} \mathrm{C}$ (cyclohexane). IR $\left(\mathrm{cm}^{-1}\right): 1660 .{ }^{1} \mathrm{H} \mathrm{NMR}\left(\mathrm{CDCl}_{3}\right) \delta: 1.54$ $\left(3 \mathrm{H}, \mathrm{d}, J=6.8 \mathrm{~Hz}, \mathrm{CH}_{3}\right), 1.71\left(3 \mathrm{H}, \mathrm{s}, \mathrm{CH}_{3}\right), 2.98(1 \mathrm{H}, \mathrm{m}, \mathrm{CH}), 3.63(1 \mathrm{H}, \mathrm{dd}, J=6.1,12.5 \mathrm{~Hz}$, $\left.\mathrm{CH}_{2}\right), 4.65\left(1 \mathrm{H}, \mathrm{dd}, J=12.8,12.7 \mathrm{~Hz}, \mathrm{CH}_{2}\right), 7.25-7.56(7 \mathrm{H}, \mathrm{m}, \mathrm{Ar}), 7.44(1 \mathrm{H}, \mathrm{s}, 1-\mathrm{CH}), 7.82$ $(2 \mathrm{H}, \mathrm{m}, \mathrm{Ar}) .{ }^{13} \mathrm{C}$ NMR $\left(\mathrm{CDCl}_{3}\right) \delta: 13.82\left(4-\mathrm{CH}_{3}\right), 22.76\left(6-\mathrm{CH}_{3}\right), 29.94(\mathrm{C}-4), 56.95(\mathrm{C}-5)$, 113.30 (C-1), 123.46, 125.05 (C-2', 6'), 126.96 (C-4'), 128.10, 128.56 (C-3',5'), 129.68, 130.29, 133.74 (C-1), 134.72 (C-6a), 135.30 (C-10a), 141.73 (C-2), 149.59 (C-3a), 170.38 (6-CO). Anal. Calcd. for $\mathrm{C}_{20} \mathrm{H}_{19} \mathrm{~N}_{3} \mathrm{O}$ (317.39): C, 75.69; H, 6.03; N, 13.24. Found: C, 75.45; H, 6.01; N, 13.27 $\%$. 
6-Acetyl-5-methyl-2-phenyl-5,6-dihydro-4H-imidazo[1,2-a][1,5]benzodiazepine (3f). White crystals, yield: $72 \%$, m.p.: $173-175{ }^{\circ} \mathrm{C}$ (cyclohexane). IR $\left(\mathrm{cm}^{-1}\right): 1650 .{ }^{1} \mathrm{H} \mathrm{NMR}\left(\mathrm{CDCl}_{3}\right) \delta: 1.29$ $\left(3 \mathrm{H}, \mathrm{d}, J=6.3 \mathrm{~Hz}, \mathrm{CH}_{3}\right), 1.67\left(3 \mathrm{H}, \mathrm{s}, 6-\mathrm{CH}_{3}\right), 2.41\left(1 \mathrm{H}, \mathrm{dd}, J=12.5,14.8 \mathrm{~Hz}, \mathrm{CH}_{2}\right), 3.22(1 \mathrm{H}$, $\left.\mathrm{dd}, J=5.8,14.8 \mathrm{~Hz}, \mathrm{CH}_{2}\right), 5.40(1 \mathrm{H}, \mathrm{m}, \mathrm{CH}), 7.25(1 \mathrm{H}, \mathrm{m}, \mathrm{H}-4), 7.29(1 \mathrm{H}, \mathrm{dd}, J=1.5,7.8 \mathrm{~Hz}$, H-7 or H-10), 7.38 (2H, m, H-3', H-5'), 7.43-7.47 (2H, m, Ar), 7.41 (1H, s, H-1), 7.54 (1H, dt, $=1.5,7.8 \mathrm{~Hz}, \mathrm{H}-9$ or H-8), $7.80(2 \mathrm{H}, \mathrm{m}, \mathrm{H}-2$ ', H-6 $) .{ }^{13} \mathrm{C} \mathrm{NMR}\left(\mathrm{CDCl}_{3}\right) \delta: 19.00\left(5-\mathrm{CH}_{3}\right), 22.98$ (6- $\left.\mathrm{CH}_{3}\right), 32.55$ (C-4), 56.05 (C-5), 113.06 (C-1), 123.32, 124.79 (C-2',6'), 126.97 (C-4'), 128.01, 128.51 (C-3',5'), 129.84, 131.81, 132.40 (C-6a), 133.41 (C-1'), 135.63 (C-10a), 141.65 (C-2), 146.46 (C-3a), 169.62 (6-CO). Anal. Calcd. for $\mathrm{C}_{20} \mathrm{H}_{19} \mathrm{~N}_{3} \mathrm{O}$ (317.39): C, 75.69; H, 6.03; N, 13.24. Found: C, 75.86; H, 6.02; N, $13.21 \%$.

\section{References}

1. Katritzky, A. R.; Abonia, R.; Yang, B.; Qi, M.; Insuasty, B. Synthesis 1998, 1487.

2. Chimirri, A.; Gitto, R.; Grasso, S.; Monforte, A.-M.; Romeo, G.; Zappala, M. Heterocycles 1993, 36, No. 3, 601.

3. Gall, M.; Kamdar, B.V. J. Org .Chem. 1981, 46, No 8, 1575.

4. Gu, Zi-Qiang; Wong, G.; Dominguez, C.; de Costa, Brian R.; Rice, K.C.; Skolnick, P. J. Med. Chem. 1993, 36, 1001.

5. Hara, T.; Itoh, K.; Itoh, N. J. Het. Chem. 1976, 13, 1233.

6. Chow, A.; Gyurik, R.; Parish, R. J. Het. Chem. 1976, 13, 163.

7. Hara, T.; Fujimori, H.; Kajama, Y.; Mori, T.; Ito, K.; Hashimoto, Y. Chem .Pharm. Bull. 1977, 25, 2584.

8. Zhang, P.; Zhang, W.; Liu, R.; Harris, B.; Skolnick, P.; Cook, J. M. J. Med. Chem. 1995, 38, 1679.

9. Liu, R.; Hu, R. J.; Zhang, P.; Skolnick, P.; Cook, J. M. J. Med. Chem. 1996, 39, 1928.

10. Grossi, G.; Di Braccio, M.; Roma, G.; Ballabeni, V.; Tognolini, M.; Calcina, F.; Barocelli, E. Eur.J. Med. Chem. 2002, 37, 933.

11. Puodziunaite, B. D.; Janciene, R.; Kosychova, L.; Stumbreviciute, Z. Arkivoc, 2000, 1(iv), 512.

12. Puodziunaite, B. A.; Yanchene, R. A.; Talaikite, Z. A.; Zaks, A. S.; Rabotnikov, Yu. M.; Usachev, E. A. Khim.-Farm.Zh. 1985, 10, 1195; Chem.Abstr. 1986, 105, 133861q. 